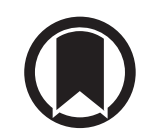

CrossMark

\title{
Age-dependent elastin degradation is enhanced in chronic obstructive pulmonary disease
}

\author{
To the Editor:
}

Chronic obstructive pulmonary disease (COPD) is primarily a lung condition characterised by the presence of persistent airflow limitation resulting from inflammation, remodelling of small airways, and emphysema. It is well-recognised that the impacts of COPD extend beyond the lung with many patients suffering systemic manifestations such as cardiovascular diseases that affect morbidity and mortality [1]. "Accelerated ageing" has been proposed as a mechanism that underlies many of the pulmonary and extrapulmonary consequences of COPD $[2,3]$. It is thought that a decline in organ function is a feature of ageing in response to the accumulation of cell and molecular damage, and in the case of COPD, noxious inhalants such as tobacco smoke increase this damage, thus accelerating the ageing process, leading to the development of COPD. With the exception of lung function decline, however, evidence indicating that tobacco smoking or COPD accelerates age-associated deterioration remains scarce.

The degradation of elastin, a key protein component of connective tissues that critically provides the characteristics of elasticity, resilience and deformability, is an important feature in normal ageing and in COPD. Elastin has a long half-life ( $\sim 74$ years [4]) in contrast to minutes to days for most intracellular proteins [5]. This longevity increases its susceptibility to oxidative and chemical damage, which are believed to drive age-related elastic fibre turnover associated with low-grade chronic inflammation. This turnover can be measured by the levels of circulating desmosine and isodesmosine (DES/IDES), two crosslinking moieties that specifically exist in mature elastin [6]. We and others have shown increased circulating DES/IDES levels in COPD patients in comparison with healthy smokers and never-smokers [7-10]. Recently, we further demonstrated that this increase was associated with higher mortality and cardiovascular morbidity in a large cohort study [10]. Interestingly, among all of the demographic variables analysed, circulating DES/IDES levels display the strongest consistent correlation with chronological age in three independent cohorts of COPD patients $[10,11]$. This marker also showed a stronger association with age than inflammatory markers (e.g. fibrinogen, club cell secretary protein 18 and high sensitivity C-reactive peptide) [10]. These observations raise the possibility that systemic elastin turnover is an age-dependent process, and that COPD and smoking can alter the rate of this process.

To test this hypothesis, we analysed data collected from three study cohorts that have been described previously [10, 11], comprising 261 never-smoker controls, 380 smoker controls and 1332 COPD patients. The relationship between circulating DES/IDES levels and age was analysed separately in each group. Consistent with the observations from previous studies [10-12], we confirmed that circulating DES/IDES levels were significantly correlated with age in all groups $(\mathrm{p}<0.0001)$ (figure $1 \mathrm{a}-\mathrm{c}$ ). The correlation was weaker in the smoker control group compared with the never-smoker control or COPD group ( $\mathrm{r}=0.41$, 0.28 , and 0.41 for never-smoker control, smoker control, and COPD groups, respectively; $\mathrm{p}<0.0001$ for all groups). The positive associations remain highly significant taking into account sex, forced expiratory volume in $1 \mathrm{~s}(\mathrm{FEV} 1)$ or pack-years smoking history $(\mathrm{p}<0.0001)$.

To investigate whether the COPD or smoker control group has a higher age-associated increase in elastin turnover, we compared the slopes of the regression lines between the three groups using a linear mixed effect model incorporating subject-specific random effect, and taking into account the effect of two visits from some individuals. We found that the slope of elastin turnover to age regression was greater in COPD patients $\left(6.6 \mathrm{ng} \cdot \mathrm{L}^{-1}\right.$ per year) compared with never-smokers $\left(2.9 \mathrm{ng} \cdot \mathrm{L}^{-1}\right.$ per year, $\left.\mathrm{p}<0.0001\right)$ or smokers (3.1 ng. $\mathrm{L}^{-1}$ per year, $\mathrm{p}<0.0001$ ) (figure $1 \mathrm{~d}$ ). Further subgroup analysis indicates that smoking status or its interaction with age in the COPD group had no significant effect on circulating DES/IDES levels $(p=0.47$ and 0.47 , respectively). In smoker control subjects, circulating DES/IDES levels were $15 \%$ higher than in never-smokers $(p=0.008)$, but no difference was found in the slope $(p=0.68)$. The difference in the intercepts between smoker controls and never-smoker controls $\left(\sim 20 \mathrm{pg} \cdot \mathrm{mL}^{-1}\right)$ suggests that smoker controls are biologically, on average, 6.9 years older than never-smoker controls.

These results strongly support the hypothesis that elastin turnover increases with age in general, and that COPD accelerates the age-dependent increase in elastin turnover. However, it does not suggest a greater 

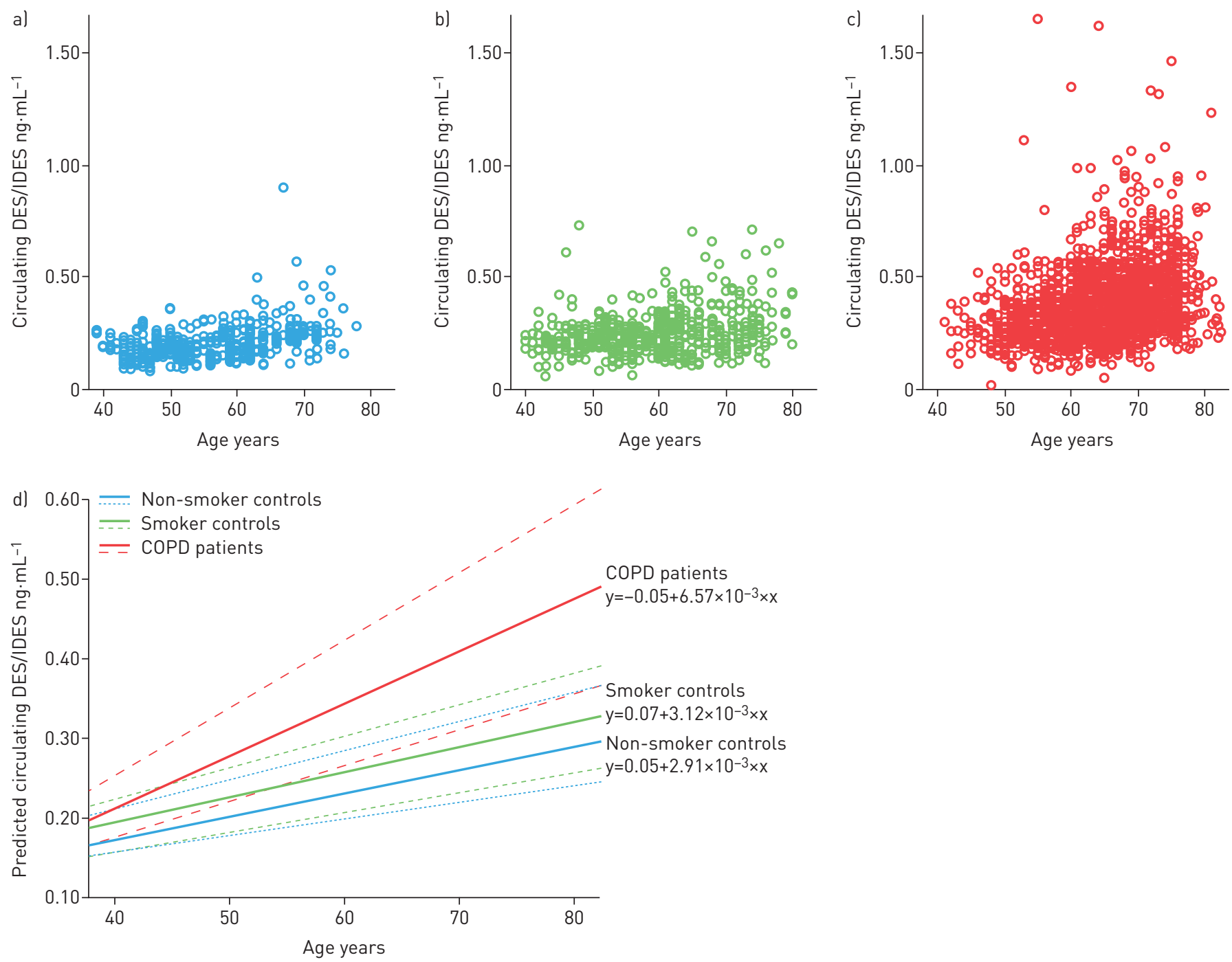

FIGURE 1 Modelling of elastin turnover as a function of age in chronic obstructive pulmonary disease (COPD) patients, smoker controls and never-smoker controls. Scatter plots of circulating desmosine and isodesmosine (DES/IDES) levels and age in a) never-smoker controls, b) smoker controls and c) patients with COPD from three cohort studies. d) Predicted linear mixed model of circulating DES/IDES levels as a function of age in never-smoker controls, smoker controls and patients with COPD. The equations of the regression lines are shown on the graph with dashed lines showing the 95\% confidence intervals. The correlation coefficients (rho) are $0.41,0.28$, and 0.41 for smoker control, never-smoker control, and COPD groups, respectively ( $p<0.0001)$.

age-related increase in smoker controls, compared with never-smokers. Overall, these results are in line with the general concept of "accelerated ageing" in COPD.

While circulating DES/IDES can come from any elastin expressing tissue, we speculate that the vascular system is the most predominant source due to its size and proximity to blood. The consequence of such elevation in elastin turnover is likely to affect the vascular physiology and cardiovascular outcomes. Indeed, we have shown that the increased circulating DES/IDES levels are associated with higher mortality and cardiovascular comorbidity in COPD [10]. The circulating DES/IDES levels did not, however, relate to emphysema progression or FEV1 decline in COPD [10], potentially due to the reasons mentioned previously.

It is worth noting that circulating DES/IDES and other biomarkers of ageing such as telomere length are different in nature, although they are all correlated with chronological age. Because circulating DES/IDES levels estimate the activity of proteolytic enzymes towards mature elastin, it may be more relevant to ageing activity at the sampling time, whereas other typical biomarkers of ageing such as telomere length may be more representative of the cumulative damage over time (i.e. biological age). It is possible that a biologically older individual can have a low ageing activity or vice versa. Thus the potential utility of these biomarkers may be different. From an intervention point of view, for example, it may be more logical to target those who are biologically young, but have higher ageing activity, than those who are biologically 
old but with low ageing activity. Conversely, it may be more sensible to measure biological age to determine long-term progression in response to an intervention.

Quantifying circulating DES/IDES may therefore represent a potential tool for monitoring normal ageing and accelerated ageing, especially in a setting of therapeutic or life-style interventions. One example comes from a study of tiotropium bromide, which was shown to reduce plasma DES/IDES levels in a small cohort of COPD patients [13]. This drug is also known to be associated with a lower probability of major and fatal cardiovascular events in a COPD population compared with the placebo group [14].

We acknowledge several limitations in the current study. First, we assume the circulating DES/IDES levels represent the rate of proteolytic enzyme activity towards mature elastin. The influence of renal excretion is not taken into account, although our previous analysis did not show an association between renal function and circulating DES/IDES levels [10]. Second, the control subjects were not followed up for long enough to observe any meaningful clinical outcomes. Third, while we did not observe an age-dependent acceleration of elastin turnover in the smoker control group, we cannot exclude the possibility of a selection bias that these smokers are more resistant to the development COPD and hence have enhanced elastin degradation with age, due to genetic or environmental factors.

In summary, we demonstrated that elastin turnover increases with age in never-smoker controls and COPD patients. The age-related amplification is enhanced in COPD but not in smokers without COPD. These results suggest that early intervention in an at-risk population should be a priority in reducing ageing-associated morbidity and mortality. Circulating DES/IDES may represent a tool to monitor the rate of ageing, particularly in identifying high-risk smokers and COPD patients.

0 @ERSpublications

Elastin turnover increases with chronological age and COPD accelerates this process beyond normal ageing http://ow.ly/7OhE301B9z9

Jeffrey T.J. Huang ${ }^{1}$, Charlotte E. Bolton ${ }^{2}$, Bruce E. Miller ${ }^{3}$, Ruth Tal-Singer ${ }^{3}$, Roberto A. Rabinovich ${ }^{4}$, Colin N.A. Palmer ${ }^{1}$, Neil C. Thomson ${ }^{5}$ and William MacNee, the Evaluation of COPD Longitudinally to Identify Predictive Surrogate Endpoints (ECLIPSE) Investigators

${ }^{1}$ School of Medicine, University of Dundee, Dundee, UK. ${ }^{2}$ Nottingham Respiratory Research Unit, School of Medicine, University of Nottingham, Nottingham, UK. ${ }^{3}$ Respiratory Therapy Area Unit, GSK, King of Prussia, PA, USA. ${ }^{4}$ MRC/

University of Edinburgh Centre for Inflammation Research, Queen's Medical Research Institute, Edinburgh, UK.

${ }^{5}$ Institute of Infection, Immunity and Inflammation, University of Glasgow, Glasgow, UK.

Correspondence: Jeffrey T.J. Huang, School of Medicine, University of Dundee, Dundee, DD1 9SY, UK

E-mail: j.t.j.huang@dundee.ac.uk

Received: April 112016 | Accepted after revision: June 092016 | First published online: Sept 012016

Clinical trial: This study is registered at clinicaltrials.gov with identifier number NCT00292552.

Support statement: The ECLIPSE study (NCT00292552, GSK Study No. SCO104960) and funding for the analysis of DES/IDES were sponsored by GSK. The Nottingham Study was supported by University of Nottingham Early Careers Research Knowledge Transfer grant and NIHR Respiratory Biomedical Research Unit (2008-2012). The Nottingham Hospitals Charity supports the Nottingham Respiratory Research Unit. Funding information for this article has been deposited with the Open Funder Registry.

Conflict of interest: Disclosures can be found alongside this article at erj.ersjournals.com

Acknowledgements: The authors would like to thank Karolina Wrobel in the Biomarker and Drug Analysis Core Facility at University of Dundee, Dundee, UK for technical support.

\section{References}

1 Calverley PM, Anderson JA, Celli B, et al. Salmeterol and fluticasone propionate and survival in chronic obstructive pulmonary disease. N Engl J Med 2007; 356: 775-789.

2 MacNee W, Rabinovich RA, Choudhury G. Ageing and the border between health and disease. Eur Respir J 2014; 44: $1332-1352$.

3 Mercado N, Ito K, Barnes PJ. Accelerated ageing of the lung in COPD: new concepts. Thorax 2015; 70: 482-489.

4 Shapiro SD, Endicott SK, Province MA, et al. Marked longevity of human lung parenchymal elastic fibers deduced from prevalence of D-aspartate and nuclear weapons-related radiocarbon. J Clin Investig 1991; 87: 1828-1834.

5 Boisvert FM, Ahmad Y, Gierliński M, et al. A quantitative spatial proteomics analysis of proteome turnover in human cells. Mol Cell Proteomics 2012; 11: M111.011429.

6 Luisetti M, Ma S, Iadarola P, et al. Desmosine as a biomarker of elastin degradation in COPD: current status and future directions. Eur Respir J 2008; 32: 1146-1157.

7 Ma S, Lieberman S, Turino GM, et al. The detection and quantitation of free desmosine and isodesmosine in human urine and their peptide-bound forms in sputum. Proc Natl Acad Sci USA 2003; 100: 12941-12943.

8 Albarbarawi $\mathrm{O}$, Barton A, Lin Z, et al. Measurement of urinary total desmosine and isodesmosine using isotope-dilution liquid chromatography-tandem mass spectrometry. Anal Chem 2010; 82: 3745-3750. 
9 Albarbarawi O, Barton A, Miller D, et al. Characterization and validation of an isotope-dilution LC-MS/MS method for quantification of total desmosine and isodesmosine in plasma and serum. Bioanalysis 2013; 5: 1991-2001.

10 Rabinovich RA, Miller BE, Wrobel K, et al. Circulating desmosine levels do not predict emphysema progression but are associated with cardiovascular risk and mortality in COPD. Eur Respir J 2016; 47: 1365-1373.

11 Huang JT, Chaudhuri R, Albarbarawi O, et al. Clinical validity of plasma and urinary desmosine as biomarkers for chronic obstructive pulmonary disease. Thorax 2012; 67: 502-508.

12 Lindberg CA, Engström G, de Verdier MG, et al. Total desmosines in plasma and urine correlate with lung function. Eur Respir J 2012; 39: 839-845.

13 Ma S, Lin YY, Tartell L, et al. The effect of tiotropium therapy on markers of elastin degradation in COPD. Respir Res 2009; 10: 12 .

14 Celli B, Decramer M, Leimer I, et al. Cardiovascular safety of tiotropium in patients with COPD. Chest 2010; 137: $20-30$.

\section{The effect of hypoxia and re-oxygenation on adipose tissue lipolysis in COPD patients}

To the Editor:

Severe chronic obstructive pulmonary disease (COPD) is a progressive disease associated with excessive mortality; however, mechanisms driving disease progression remain unclear [1]. A pro-inflammatory state, metabolic impairments and respiratory muscle weakness contribute to deterioration of lung function $[1,2]$. Furthermore, a poor COPD prognosis appears to be associated with unintentional weight loss [3, 4], which has been observed in $\sim 50 \%$ of severe COPD patients [5].

Adipose tissue secretion of pro-inflammatory substances is modified by tissue $\mathrm{O}_{2}$ levels [6]. Transient drops in blood oxygenation dropping to arterial oxygen saturation measured by pulse oximetry $\left(\mathrm{SpO}_{2}\right)$ levels of $60-70 \%$ have been shown to induce profound adipose tissue hypoxia with oxygen tension $\left(\mathrm{PO}_{2}\right)$ levels as low as $30-40 \mathrm{mmHg}$ [7], which resemble levels typically observed in obesity and which are associated with augmented lipolysis and elevated levels of circulating free fatty acids (FFA) [8, 9]. In fact, elevated plasma FFA levels in COPD patients [10] could be due to increased adipose tissue lipolysis. Considering that chronic exposure to higher plasma FFA concentrations can lead to lipotoxicity in cardiac muscle and impaired energy metabolism in skeletal (respiratory) muscle, it can be hypothesised that enhanced lipolysis worsens the COPD prognosis through its negative impact on respiratory muscle function, progression of right heart failure, and development of cachexia.

Regulation of adipose tissue lipolysis in COPD patients is potentially associated with adipose tissue hypoxia, activation of the sympathetic autonomic nervous system and/or elevation of pro-inflammatory cytokines [11, 12]. Additionally, elevated plasma levels of lipolysis-stimulating natriuretic peptides (atrial natriuretic peptide (ANP) and brain natriuretic peptide (BNP)) have been found in COPD patients, which provides an additional mechanism for enhanced lipolysis [13].

The aim of this study was to investigate the lipolytic response, of adipose tissue, to acute changes in arterial oxygen levels and to assess the role of acute ANP and catecholamine administration in a group of severe COPD patients.

Eight subjects (four men, aged 61.4 \pm 2.8 years, body mass index (BMI) $24.4 \pm 0.8 \mathrm{~kg} \cdot \mathrm{m}^{-2}$ ) with severe COPD (GOLD IV category diagnostic criteria) who had been prescribed long term oxygen therapy (LTOT) $>1$ year were recruited. Exclusion criteria included acute exacerbation of COPD, other acute or decompensated illnesses (inflammatory, endocrine), unstable body weight over the 3 months prior to entering the study, age $>75$ years and cancer. All subjects gave written informed consent and the study was approved by the Ethics Committee of the Third Faculty of Medicine, Charles University in Prague.

Two microdialysis probes (20.000 Da cut-off, CMA Microdialysis, Kista, Sweden) were inserted into the subcutaneous abdominal adipose tissue. The probes were perfused with Ringer's solution (Baxter, Prague, Czech Republic) supplemented with $1.7 \mathrm{~g} \cdot \mathrm{L}^{-1}$ ethanol with or without $10^{-6} \mathrm{~mol} \cdot \mathrm{L}^{-1}$ ANP or $10^{-6} \mathrm{~mol} \cdot \mathrm{L}^{-1}$ adrenaline. The outflowing dialysate was collected for glycerol (Randox Laboratories, Crumlin, UK), ethanol (BioVision, Milpitas, CA, USA) and urea (Abcam, Cambridge, UK) quantification. The dialysate glycerol concentration served as a marker of lipolysis, while the ethanol inflow/outflow ratio and urea 